\title{
25. COMMISSION DE PHOTOMETRIE STELLAIRE
}

\section{Reports of Meetings}

President: V. B. Nikonov.

Secretary: D. L. Crawford.

\section{Business Meeting, 26 August 1964}

Dr Nikonov opened the meeting of the Commission, at $9^{\mathrm{h}} 00^{\mathrm{m}}$, by welcoming all members and guests and asked that those present stand for a moment in memory of the two Commission members who died since the last meeting.

The following Organizing Committee was agreed upon: President, V. B. Nikonov; VicePresident, A. W. J. Cousins; Organizing Committee: D. L. Crawford, H. Haffner, R. H. Hardie, G. E. Kron, K. Osawa, E. Rybka, and A. G. P. Velghe.

\section{DRAFT REPORT}

The Draft Report was adopted with only a few corrections, mostly misprints. A number of additions were mentioned. Crawford noted that now in operation at Kitt Peak Observatory were two I6-inch telescopes, one 36 -inch telescope, and one 84 -inch telescope. All are equipped with standard photoelectric photometers. A second 36 -inch telescope is now under construction and a 150 -inch telescope is being designed. Nikonov pointed out that there are undoubtedly some references not included in the bibliography and some important references were published after the bibliography was made up, in particular the Basic Astronomical Data volume. The group felt that the report was very good, especially the references.

\section{REPORTS AND DISCUSSIONS}

Nikonov raised the question of the scope of this Commission. He felt that much work is being duplicated in other Commissions and proposed that Commission 25 restrict its area of competence. Photometric units, methods of reductions, standards, and so forth should definitely be a major part of Commission 25, in particular all standards and standard sequences should be kept under the Commission. Photometry done above the atmosphere, and in any wavelength regions, should, of course, be included. It should not be necessary to include applications of photometry. He noted that this proposal had been discussed by the Organizing Committee, and they all felt that such a restriction would be most useful.

Nikonov then listed the areas that might well be included: I. Photometric instrumentation, 2. methods of photometric observing and reduction, 3. extinction, 4. photometric systems (all band widths), 5. photometric standards, and 6 . photometric sequences (secondary standards from all sources). These items were briefly discussed, and it was felt that this scope would be very good and was of sufficient size for this Commission.

$R y b k a$ stressed extinction problems and noted that differential extinction is important, especially where climates are poor. He remarked on the zero point network reported in previous IAU sessions. This work is now finished, the magnitudes of pairs of stars of sixth magnitude near Selected Areas are ready and may serve for zero point and extinction determinations.

Hogg noted that the above items implicitly include polarization. Hoag felt that a statement concerning intrinsic stability of stars should be included under standards. Kron felt that 
including photometric instrumentation in Commission 25 was important, for many of the instruments are specialized and apply only to photometry. Behr questioned whether image tubes should be included, and the discussion result was that probably they should not be. Commission 9 has a special Sub-Committee on Image Tubes, for example.

Steinlin felt that a serious question which should be discussed at this meeting of the IAU is the matter of photographic material. During the last years it has become more and more difficult to obtain suitable photographic material from the manufacturers. Either the production of certain emulsions is completely stopped or prices have gone up tremendously or very large minimum orders have to be given that are far higher than any one observatory can afford or use. It should be carefully considered whether there is not a possibility that the IAU can arrange for a common effort or pool of observatories for:

(I) Common ordering or other organization of furnishing plates to observatories which need only small amounts of plates or of special emulsions.

(2) Investigating the properties of plates of preferably more than one firm to insure delivery of plates of good and constant qualities. It is not quite clear which Commission of the Union is actually the one which should deal with this matter, but it is certainly a matter of great importance for this Commission.

Other Commissions are interested in this same problem, and perhaps Commission 9 may even propose a resolution to the Executive Committee.

Sinvhal asked if anyone could express an opinion, based on experience, on the astronomical emulsions put out by Agfa of East Germany? Dr Haffner answered: Their blue sensitive emulsions are comparable to Kodak I03a-O, but their red emulsions are considerably less sensitive than Kodak 103a-E.

Several people felt that a central clearing house which could order and test plates for many observatories would be welcome.

Willstrop felt that the Commission should be concerned under item 5 with the relationship between stellar magnitudes and c.g.s. units of radiation. Visiting the Radcliffe Observatory in July-September 1962, R. V. Willstrop (Cambridge Observatories) made spectral scans of 215 stars at $5 \circ \AA$ resolution. The scans have been normalized using observations made at the Cape of Good Hope (Mon. Not. R. astr. Soc., I21, 17, I960) to determine the flux received outside the Earth's atmosphere from stars of apparent magnitude V=0.00 (Mem.R. astr. Soc., in press, 1964).

Nikonov then proposed that the Commission adopt this framework from now on, and the Commission members agreed.

The Canadian IAU committee suggested that Commission 25 propose that all colour magnitude diagrams have the same scale with units of I magnitude in $V$ and 0.2 magnitudes in colour index. Following discussion, the Commission felt that Commission 37 should make such proposals.

\section{Scientific Meeting, 28 August 1964}

\section{SCIENTIFIC REPORTS}

Dr Behr discussed the results of his study of temperature effects and cathode sensitivity in photomultipliers. His tests were on the RCA 6I99 and 7102, and the EMI 6r94. He found that from the maximum sensitivity wavelength to the red wavelengths, the sensitivity curve shifts parallel by up to $5 \%$ for dry ice temperatures. The change is about linear with temperature. A temperature change of about $10^{\circ} \mathrm{C}$ will give a difference of $\circ^{m} \cdot{ }^{\circ 2}$ in $(B-V)$. $\mathrm{He}$ found also that the sensitivity of the surface drops steeply towards the edge of the cathode and 
that the maximum sensitivity is not in the geometric centre. A shift of about $0.0 \mathrm{I} \mathrm{mm}$ could cause errors of $0^{m} .02$. The sensitivity pattern may be different for different colours. Part of the variation is due to collection efficiency and not just cathode sensitivity. The 7102's vary a lot among themselves, especially at the red end. The 6rog's that are most noisy are not astronomically useful, but those with low dark currents are useful at higher light levels. The I P2 I still appears to be the best available photomultiplier (except in the red, of course).

$\mathrm{Dr}$ Behr then described his 4-channel polarimeter, which is used to determine the three Stokes parameters. The system has been designed primarily for extended sources, and the output is fully digitized through DC amplifiers and voltage to frequency converters. The light of the source is measured simultaneously in four parallel telescopes, each having an analyser at a different plane of vibration. The system has been described in Sonderdruck aus Sitzungsberichte der Heidelberger Akademie der Wissenschaften, Jahrgang 1962-3, 2. Abhandlung, S. 85-90'.

Crawford described briefly the $\mathrm{H} \beta$ and 4 -colour systems in use at Kitt Peak. An extended list of standard stars defining the $\beta$ system will go to press shortly. It will include stars of spectral type Go and earlier. The Strömgren 4-colour system (described in the Basic Astronomical Data volume) is being extended to all spectral types at Kitt Peak. These systems were described in a paper given by Crawford at the IAU Symposium no. 24 on Spectral Classifications held at the Stockholm Observatory.

Dr Hoag reviewed a proposal from Dr Ejnar Hertzsprung. Professor Hertzsprung, in communication with staff members of the U.S. Naval Observatory, has proposed that a network of 92 photometric standard stars be established so that no object in the sky will be more than $12^{\circ}$ away from any of three standards. He further proposes that all standard stars be of class $F$ or earlier; between 7 th and 8th magnitude; and be free from effects of duplicity and variability. Dr Hertzsprung proposes that the observations be made photoelectrically with narrow band filters centred at $425^{\circ}, 55^{\circ}$ and $825^{\circ} \AA$.

Professor Hertzsprung has provided co-ordinates for centres fulfilling the requirements of the proposed network together with a list of 1809 stars, chosen from the Henry Draper Catalogue, from which selections of appropriate standards could be made.

Hoag noted, in transmitting the proposal, that the wavelength bands had been selected to be relatively free of line blanketing effects. The $8250 \AA$ region has been selected to avoid telluric water bands as well as emission lines that occur in $\mathrm{H}$ II regions and some stars. Of the three bands, only the $5500 \AA$ region corresponds to systems in current usage. The colours based on the proposed system would not be particularly sensitive to luminosity effects in early type stars and the band at $8250 \AA$ would not be suitable for photoelectric measures of faint stars with currently available receivers and moderate sized telescopes. Every effort, however, should be made to continue photometric measures at long wavelengths.

Dr Bertiau noted that he has a computer programme in use at Vatican Observatory that may be used to get the best solution for photometry of stars on new systems (no available standard values). All nights are solved together, but each night is allowed to have separate zero points and extinction coefficients. A discussion followed concerning the variability of extinction over a given night, an effect which is certainly present at many observatories. Some observers have noted that the colour term in extinction varies as well. At the University of Michigan, Dr Boyce found variabilities in this term of plus or minus $20 \%$ over the course of a year from his scanner observations.

A number of observers remarked on the extinction problems in the southern hemisphere after the eruption of Mt Agung. Increases in the visual extinction of up to four times normal values were noted. The extinction appeared to be neutral. 


\section{DISCUSSION OF A STANDARD MULTICOLOUR SYSTEM}

Nikonov raised the question of whether this Commission should now propose, or at least begin discussing, a possible standard multicolour system and whether a sub-committee should be formed to work jointly with the new Commission 45 concerning such a system. The problem is a difficult one, but the hope would be that the best system could be chosen on the basis of available data. It would probably use intermediate band widths similar to some of the systems now in use. One should perhaps choose such a system by tests (on a computer) of different filter curves combined with stellar energy curves, interstellar reddening curves, and atmospheric extinction curves.

Nikonov then read a paper by V. Straižys which may be summarized as follows:

\section{ON THE SEARCH FOR AN OPTIMUM MULTICOLOUR PHOTOMETRIC SYSTEM FOR STELLAR ASTRONOMY \\ V. Straizys}

(Vilnius Astronomical Observatory, U.S.S.R.)

The optimum multicolour photometric system should allow the most accurate determination by photometric measurements only of the following characteristics of stars and of interstellar matter: (I) spectral type, (2) luminosity class, (3) population type, (4) interstellar absorption. None of the current photometric systems is able to settle this problem for all types of stars without prior knowledge of the spectral type. Several authors recently discussed their efforts on new multicolour photometric systems $(\mathbf{I}-3)$. The work at Vilnius Observatory is based on stellar energy distribution data published by different authors, and assorted filter transmission curves of different wavelengths and band widths. Whitford's interstellar absorption curve is being used. The computations are being carried out with a high-speed electronic computer of the BESM-2 type. Some of the first results of this work are considered in the present report.

The band widths of response curves of the new photometric system must satisfy the following requirements: the band width effects must be negligible, but the limiting magnit ude must be sufficient. When varying the band widths of the response curves of $U, B, V$ system, it can be shown that a considerable degree of constancy of the well known parameters $r$ and $R$, defined by the equations:

$$
\begin{gathered}
\frac{E_{(U-B)}}{E_{(B-V)}}=r+S E_{(B-V)} \\
R=\frac{A_{V}}{E_{(B-V)}}
\end{gathered}
$$

is attained when the half-width of standard bands has been reduced to $200 \AA(5,6)$. At this width, the range of variation of $r$ and $R$ is only few percent of the corresponding variation in the original system $U, B, V$. The curvature of reddening-lines practically disappears when the band widths are of order of $400 \AA$.

So the half-widths of response curves of the new photometric system must not exceed 200-250 $\AA$, and this can be realized only by means of appropriate interference filters.

A question of great importance is whether a three-colour system permits the determination of the interstellar reddening from a two-colour diagram without the knowledge of spectral classes $(\mathbf{x}, \mathbf{9}, \mathbf{1 7})$. The line of unreddened stars in an ideal diagram must satisfy the following conditions: ( $r$ ) the angle between this line and the reddened one should be as large as possible, (2) this line should not have considerable bend or curvature, especially in the direction of 
reddening lines, (3) the unreddened line should coincide for unreddened stars of all luminosities and populations.

Unfortunately, such a system cannot be found. When two first conditions are realized, the third one never takes place (ro, Ix). So it is necessary to use other magnitudes for the determination of spectral and luminosity classes without knowledge of interstellar reddening. The $Q$-method, first used by Professor Becker (12) and developed in the well-known work of Johnson and Morgan (13) gives the necessary solution if the magnitudes are being measured in appropriate spectral regions and the law of reddening is determined.

Let us suppose that spectral, luminosity, and population types of stars are known and their colour-excesses are to be determined. Then the third of the above mentioned conditions falls out, and we shall try to find a three colour system satisfying only the first two ones. Such a system consists of the following magnitudes: (I) magnitude $X$, placed on the short wavelength side of the 'break' point of interstellar absorption curve, but above the Balmer jump $(\lambda=3900-4200 \AA)$, (2) magnitude $Y$, placed at the mentioned 'break' at $\lambda=4500 \AA$, (3) magnitude $Z$, placed on the red side of the 'break'. In one of the author's papers (I), the following preliminary mean wavelengths were selected: $\bar{\lambda}(X)=4000 \AA, \bar{\lambda}(Y)=4500 \AA$. It was necessary to find more accurate positions of $X$ and $Y$ and investigate how the position of magnitude $Z$ influences the diagram $(X-Y),(Y-Z)$.

The computations were carried out using stellar energy curves of Code (14) and Melbourne (15), in which absorption lines with $W \geqslant \mathrm{I} \AA$ were taken into account. Criteria of the quality of the diagram $(X-Y),(Y-Z)$ were: (I) the curvature of the main-sequence line at Ao $\mathrm{V}$, characterized by the angle $\alpha$ (the angle between the line from Bo to Ao and the line from Bo to Fo); (2) the difference in directions of spectral and interstellar reddening, characterized by the ratio of slope of the line Bo - Fo and the slope of reddening line:

$$
n=\frac{(X-Y)_{\mathrm{Fo}}-(X-Y)_{\mathrm{Yo}}}{(Y-Z)_{\mathrm{Fo}}-(Y-Z)_{\mathrm{BO}}} \times \frac{E_{(Y-Z)}}{E_{(X-Y)}} .
$$

The mean wavelength of magnitude $X$ was changed by increments of $5^{\circ} \AA$ from $3900 \AA$ to $4200 \AA$ in the computations. The corresponding variation of $\bar{\lambda}$ for magnitude $Y$ was from $4300 \AA$ to $4700 \AA$ and for magnitude $Z$ from 4800 to $6600 \AA$. The band widths of rectangular and interference filter-like transmission curves were changed from $25^{\circ} \AA$ to $400 \AA$. Some results of this investigation are given below.

(I) The curvature of the main-sequence line in the diagram $(X-Y),(Y-Z)$ in the vicinity of Ao $\mathrm{V}$ depends on the combination of positions of all three filter curves. Changing the third $\bar{\lambda}$, one can make the angle $\alpha$ positive or negative. A magnitude of this angle $\alpha$ varies slightly with the band width and with the form of response curves.

(2) The position of the $Y$ response curve, determined preliminarily, must be shifted towards the longer waves to avoid the influence of $\mathrm{H} \gamma$ line and the interstellar band at $443 \circ \AA$. However, this shift does not exceed $4600 \AA$ because of the corresponding diminution of the angle between the main-sequence and reddening lines.

(3) The ratio of the slopes of the main-sequence and reddening lines, ' $n$ ', varies with the positions of all the filters. It would depend upon the position of $\bar{\lambda}(Y)$ only if the law of temperature reddening of stars were exactly $\mathrm{r} / \lambda$. Then the maximum value of $n$ would be reached at the coincidence of $\bar{\lambda}(Y)$ with the break point of interstellar reddening curve. The departures of temperature reddening from $\mathrm{I} / \lambda$ law causes the dependence of $n$ to act in the following way: (I) when the positions of $\bar{\lambda}(X)$ and $\bar{\lambda}(Y)$ are fixed, and only $\bar{\lambda}(Z)$ varies, the highest value of $n$ is reached at $\bar{\lambda}(Z)-5300 \AA,(2)$ when the positions of $\bar{\lambda}(Y)$ and $\bar{\lambda}(Z)$ are fixed and only $\bar{\lambda}(X)$ varies the ratio $n$ strongly increases when $\bar{\lambda}(X)$ diminishes. The system in which the slope of 
main-sequence line exceeds by factor of 2.8 the slope of reddening line may be realized by corresponding selection of $\bar{\lambda}$ of all three magnitudes.

However, the diminution of $\bar{\lambda}(X)$ increases not only the ratio $n$ but also the curvature of the main-sequence line, and it becomes impossible to straighten it by selection of $\bar{\lambda}(Y)$ and $\bar{\lambda}(Z)$. Nevertheless, the gain in ratio $n$ is so great that the curvature becomes not so harmful and only slightly diminishes the precision of determination of colour excesses. The two-colour diagram $(X-Y)(Y-Z)$ for the system with $\bar{\lambda}=3900,4600$, and $5300 \AA$ resembles the Borgman diagram $(N-M)(M-L)(\mathbf{1 6})$, but the angle between the main-sequence and reddening line is considerably greater. The $(X-Y)(Y-Z)$ type of two-colour diagrams have an interesting property: the line of unreddened supergiants intersects the main-sequence line at spectral class Go. One can separate the regions of stars earlier and later than Go by the reddening line at Go. Unfortunately, this diagram does not separate luminosities.

The well known Borgman (16) two-colour diagram $(Q-P)(P-N)(\lambda(Q)=3560 \AA, \lambda(P)$ $=375 \circ \AA$, and $\lambda(N)=4055 \AA)$ supplements the diagram $(X-Y)(Y-Z)$. In the twocolour diagram $(Q-P)(P-N)$ the reddening line of Bo V star entirely separates the mainsequence stars from supergiants of type $B, A$, and $F$. One can determine the luminosity and spectral classes for the stars earlier than Go by a combination of two-colour diagrams $(X-Y)$ $(Y-Z)$ and a two $Q$ index diagram analogous to the similar Borgman diagram $(\alpha, \epsilon)$. The only difference is the replacement of Borgman's $Q_{N M L}$ by $Q_{X Y Z}$.

Thus, the five-colour system $Q, P, X, Y, Z$ permits two dimensional spectral classification and a single valued determination of interstellar absorption for stars earlier than $\mathrm{F}_{5}$ or Go.

The problems to be solved now are the following: selection of more accurate positions of the magnitudes considered above: photometric classifications of spectral and luminosity classification of late-type stars and determination of criteria of stellar chemical composition. Let us hope that the number of magnitudes necessary for this task will not become too large.

\section{REFERENCES}

I. Straižys, V. Bull. Vilnius Obs., no. 6, I, I963.

2. Becker, W. in Stars and Stellar Systems, Vol. III : Basic Astronomical Data. Ed. K. Aa. Strand. Univ. Chicago Press, 1963, p. 24r.

3. Steinlin, U. Contr. Bosscha Obs., no. 21, 34, I963.

4. Whitford, A. E. Astr. $\mathcal{~} ., 63,201,1958$.

5. Straižys, V., Zdanavičus, K. Astr. Zu., 4I, 5 19, 1964.

6. Straižys, V., Zdanavičius, K. Astr. Cirk. no. 254, 5, 1963.

7. Golay, M. Publ. Obs. Genève, fasc. 64, 1963.

8. Borgman, J. Bull. astr. Inst. Netherlds, 15, 255, 1960.

9. Straižys, V. Astr. Cirk. no. 254, 3, 1963.

го. Straižys, V., Zdanavičius, K. Abastumanskaja astrof. Obs. Bjull., 1964 (in press).

rr. Zdanavičius, K., Straižys, V. Bull. Vilnius Obs., no. I r, I964 (in press).

12. Becker, W. Z. Astrophys., 15, 225, I938.

13. Johnson, H. L., Morgan, W. W. Astrophys. F., 117, 313, I953.

14. Code, A. D. in Stars and Stellar Systems, Vol. VI: Stellar Atmospheres. Ed. J. L. Greenstein. Univ. Chicago Press, 1960, p. 50.

15. Melbourne, W. G. Astrophys. F., 132, 101, 1960.

16. Borgman, J. Bull. astr. Inst. Netherlds, 17, 58, 1963.

17. Straižys, V. Astr. $Z u$., 41, 750, 1964.

\section{DISCUSSION}

Dr Becker stated that an optimum system is an ideal: all real systems must be compromises. The system that one uses will depend strongly on the application. For example, in high 
galactic latitudes there is little interstellar reddening and supergiants are very rare. Perhaps the best system there should separate luminosity and population effects. A simple rule would be to have the ultraviolet effects in one filter and no ultraviolet effects in the others.

In addition to Dr Becker's remarks, Steinlin also mentioned that he would like to emphasize that he thought that there cannot be one single 'best multicolour system', above all in view of the many very different applications such systems are used for. One has to think of the wide array of tasks which it has to fulfil: cluster work, differentiation of different spectral classes, of luminosity classes, of different metal contents and population groups, determination of interstellar reddening and so on. So it is highly improbable that one and only one ideal system can fulfil all these tasks at its best. Instead of searching for one such system one should first state the problem to be solved and then go and find the system that is best suited to fulfil this task. For different purposes the requirements may be contradictory and we shall need different systems. There is, for instance, the question of the band-width: for many purposes one wishes bands as small as necessary to avoid all second order terms of temperature effects, interstellar and atmospheric reddening; on the other hand, for work with very faint stars one prefers to have broader bands to gain one or two magnitudes even when one has to cope with these difficulties. There is not even a common opinion on the number of colours one should have in a standard system: in general, one speaks about three colour photometry, but just now we heard about a very gratifying system of four colours. One more point to consider is whether the system should serve for photographic or photoelectric work: there are today very good possibilities to set up a photoelectric system with interference filters, but it is impossible to have such filters in the necessary size to use them in Schmidt telescopes or astrographs for photographic work.

All this should show that it is not correct to search for one and only one "best photometric system'. This could not be anything else but a compromise of of ten contradicting requirements for different purposes and would therefore fulfil none of them in the best way. In the end observers would still prefer to use another system that fulfils their special task better than this standard system.

Dr Seittler agreed, but felt that the system should be checked by computation. She has a computer programme that will use stellar energy curves (including line effects) obtained from objective prism plates (with $\mathrm{IN}$ emulsions). These curves agree well with Oke's scanner results. Not only the location and band width of the filter is important, but its shape as well. Oke remarked that a study of 25 Hyades stars is about finished, with a $\Delta \lambda$ of $5^{\circ} \AA$.

McCarthy commented that the choice of an infra-red point for colour work is very important, and that he would like to urge members to pay attention to the range covered by the Kodak IN plates, which in connection with Wratten 89 filter or a $R_{5}$ filter will isolate a region from $6800 \AA$ to $8800 \AA$ approximately. Kron and his co-workers have observed many stars on the $R-I$ system and these will be available for photographic interpolation from photoelectric sequences. As new multialkali cells begin to become available, care should be taken to choose proper red and infra-red points that will be compatible with the Kodak IN plates. Care must be taken to avoid the troublesome terrestrial bands of watervapour.

Steinlin felt that it may be possible to have a general agreement on the choice of colours in this part of the spectrum. If no effort is made soon, we will soon have the confusion of many different systems. Here is a chance to show how to undertake a search for a really good system and to attempt a general agreement on the adoption of a colour system. It is time to do so above all in view of the start of extraterrestrial observations, which require a system of colours adopted to astrophysical needs and not available filters, watervapour bands, etc. Bidelman raised the important point that there is a strong reason for some standard system, however, for astronomers still need magnitudes and colours for statistical use, and many spectroscopists depend strongly 
on such uniform systems. Crawford remarked that a pending programme at Kitt Peak is the measurement of all stars to about 8.5 magnitude on some system, which is yet unspecified. Stoy proposed that at least we can agree on one magnitude system, and that it probably should be the $V$ one.

With respect to the rapid progress of the extraterrestrial observational technique, Loden feels it reasonable to choose a new wavelength regions system not too much with consideration to present terrestrial observational limitations. If, for instance, a physically very suitable colour region is found in the infrared, there should not be a too serious objection if this region coincides with an atmospheric absorption region. $\mathrm{Dr}$ I. Alma'r (Komkoly Observatory, Hungary) noted also that if there is a list of suitably chosen standard stars, then it would be valuable to secure a series of measures of them by means of an OAO satellite.

Nikonov said that there seems now to be many multicolour systems. In wide band photometry the $R G U$ is very good, and there are many standard photoelectric observations on the $U B V$ system and this system is widely used. It may turn out that the most used multicolour system will become the standard one, and therefore one must think seriously now about which system to use on large scale programme. He then proposed that a joint working group be formed to investigate the problems of a multicolour standard system and to prepare a statement for the next Commission and General Assembly meetings. The Commission approved and left it to the President to appoint such a group.

Golay believed that when investigating photometric systems one should evaluate the new systems by studying high dispersion spectra. One can study the energy curves then for small wavelength regions. This problem certainly concerns Commissions $3^{6}$ and 29. It will be necessary to establish a list of stars to be studied, and the stars could then be measured with the proposed photometric systems. Normal stars of each spectral type must be included, as well as certain special stars, such as metallic line stars and sub-dwarfs. Spectroscopic double stars should also be included to study their effects on the system.

Hoag noted that the whole history of systems so far has depended greatly on the receivers and not on astrophysical choices. Steinlin felt that even if one cannot recommend one single three- or four-colour system, at least a list of a greater number of colours or colour regions over the whole range of the spectrum will allow an observer to choose different combinations of colours for different types of investigations. It is perhaps useful to have a system of six or eight colours (and standard stars measured in all these colours) and to have recommendations perhaps in the following form: for this specific purpose use colours 1,4 and 7 , for that other purpose use colours 2,4 , and 6 , etc. This leaves room for the different requirements and still gives a possibility of a certain standardization.

\section{Organizing Committee Meeting, I September r964}

Present: Nikonov, Crawford, Haffner, Rybka, Velghe.

(I) New Members proposed by National Committees: Bahng, U.S.A.; Gallouët, France; Jackisch, German Democratic Republic; Moreno, Chile; Pesch, U.S.A. The Organizing Committee believed that Steinlin should also be added, and agreed that all these new members should be accepted.

(2) Changes in Organizing Committee: Ramberg requested that his name be removed as a member of the Organizing Committee, and Crawford was added.

(3) Nikonov will ask by letter if present members wish to remain members since the scope of the Commission has been changed somewhat. 
(4) The work on the next Draft Report will be divided tentatively as follows: (A) Instrumentation: Kron, Nikonov; (B) Methods of Observation and Extinction: Hardie and Nikonov; (C) Reductions: Hardie, Osawa; (D) Standards: Rybka, Haffner; (E) Systems: Cousins, Crawford; (F) Polarization: Velghe; and (G) Outside Atmospheric Observations: Velghe. Members will be asked to send their reports and bibliographies to the above by October 1966 .

(5) Nikonov appointed a tentative Working Group on Multicolour Photometric Systems as follows: Nikonov, President; Members: Borgman, Crawford, Golay, Kron, Masani, Mianes, Seittler, Strömgren, and Walraven. 